\title{
SPÖ́TLICHT
}

ON YOGA RESEARCH

November 2020

DOI: $10.35831 /$ soyr/ps2020

\section{Cardio-Pulmonary Physiology during Yoga Inversion Practice}

Paula Pullen (aka Seffens) ${ }^{1}$ and William Seffens ${ }^{2}$

${ }^{1}$ Department of Kinesiology, University of North Georgia, Gainesville, GA USA, (paula.seffens@ung.edu)

${ }^{2}$ Seftec, Inc. Atlanta, GA USA

\begin{abstract}
Introduction: Mass media advertisements have claimed health benefits of body inversion for relaxation and cardiovascular conditioning. We conducted a preliminary study to evaluate real time physiological changes and responses to mediation, Hatha yoga, and specifically inversion and standing postures to determine the $\mathrm{O}_{2}$ consumption recorded by a wearable metabolic device and cardiovascular measures. Methods: Healthy study volunteers executed a sequence of yoga postures that included inversions of whole body while wearing a Cosmed K5 portable metabolic backpack. We obtained brachial blood pressure during the last 30 seconds of each posture. Each trial began seated, followed by a warm-up consisting of gentle flow yoga and ending with relaxation. Results: Twelve experienced yoga practitioners (mean age 44 years, 58\% female) participated in 17 trials. Over all trials, mean $\mathrm{VO}_{2}$ for Sirsasana as compared with the supported inversion posture decreased from 8.4 to 4.9 ( $\mathrm{ml} / \mathrm{kg} / \mathrm{min}$ ). Conclusions: Conflicting findings exist in the literature concerning inversion physiology. Cardiac output response to inversion is not consistent in scientific reports. Participants responded differently under a variety of circumstances in previous studies, making comparisons to this and existing research challenging. We find sufficient cause for further research and suggest that some forms of inversion may be beneficial to heart failure patients.
\end{abstract}

Keywords: yoga, inversion, cardiopulmonary, heart failure, physiology 


\section{Introduction}

Recent advertisements have claimed health benefits of body inversion for stress reduction and cardiovascular conditioning in mass media (Yoshikawa, 2017). Inversion and head-down tilt were initially introduced as laboratory methods for studying physiologic changes, utilized to simulate weightlessness in space (Blomqvist et al., 1980). Historically, inversion has been widely used as a therapeutic modality to resolve musculoskeletal back pain (deVries \& Cailliet, 1985). Inversion while performing vertical headdown sit-up exercises at various degrees was reported to effect the strengthening of abdominal muscles (Klatz, 1983; Boone \& Johns, 1989).

Several studies have examined inversion therapy for a variety of cardiopulmonary disorders, including pulmonary emphysema, hypertension, coronary artery disease, and heart failure (Alvan et al., 1954; Klatz et al., 1985; Yadav et al., 2015; Pullen et al., 2018). We conducted a preliminary study to evaluate real time physiological changes and responses to meditation, Hatha yoga, inversion postures and standing postures to determine oxygen consumption $\left(\mathrm{VO}_{2}\right)$ and other cardiopulmonary variables utilizing a wearable metabolic device (Conroy et al., 2019). This effort was to evaluate the appropriateness of inversion with heart failure (HF) patients, requiring a physical regime characterized by low $\mathrm{VO}_{2}$ and low impact (Pullen et al., 2010).

\section{Oxygen Consumption and Yoga}

Hatha yoga is one style of yogic practice that traditionally lasts up to 90 minutes and typically involves a series of asanas (poses or postures) with Pranayama (breath control/exercises) and Savasana (corpse pose/relaxation). Depending on the style of yoga, studio, or teacher, a yoga session typically involves standing, seated, or supine asanas held for various amounts of time. The practice of yoga often involves balance and inversion poses with focus on proper form, alignment, and breathing. Yoga has emerged in new-generation active computer games called exergames with Microsoft Kinect and Wii Fit software platforms (Pullen \& Seffens, 2018). Energy cost as $\mathrm{O}_{2}$ consumed per kg body mass per minute 
and metabolic equivalents (METs, where $1 \mathrm{MET} \sim 3.5 \mathrm{ml} / \mathrm{kg} / \mathrm{min}$ for average resting $\mathrm{O}_{2}$ consumption) of individual yoga asanas has recently been systematically reviewed (Larson-Meyer, 2016). Eight studies found in the literature examined the metabolic cost of several yoga asanas held in traditional yoga

Figure 1.

Portable metabolic K5 measurements during yoga.

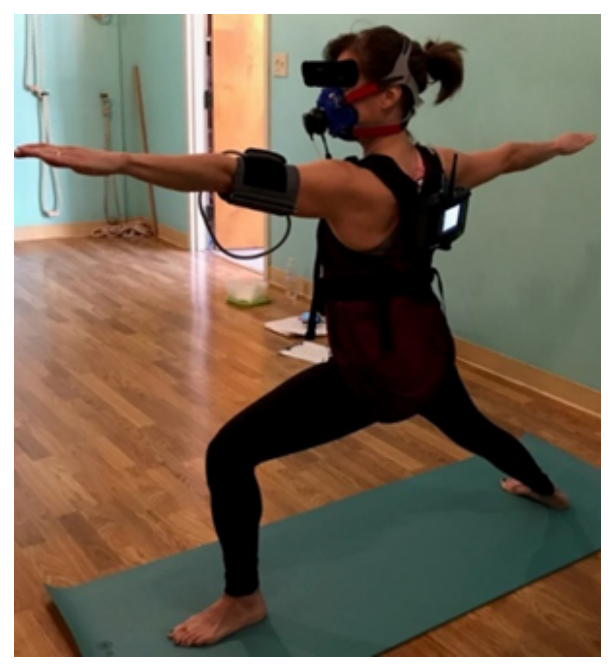

postures. Standing asanas displayed slightly higher average MET values than seated poses. Average METs of supine poses (i.e., Savasana or corpse pose) were similar to average resting values (MET value at rest $=1)$. Inversion poses, including Sirsasana (headstand) did not increase metabolic rate higher than 1.7 to 2.5 METs. Metabolic cost of some of the more difficult inversions/arm balancing poses, including Bakasana (crow/crane), Eka Pada Galavasana (flying pigeon), and Adho Mukha Vrksasana (handstand), produced MET values $>3$ (ibid.).

\section{Physiology of Inversion}

Sympathetic and parasympathetic nervous system (PNS) impulses balance the cardiopulmonary response and blood flow when changing from standing to supine body positions (Birjiniuk, \& Heldt, 2019). Hydrostatic pressure of a liquid is greater at the base of a column as the liquid above weight increases. This implies when standing in upright position, blood pressure (BP) tends to be greater in the feet than in the head. Hydrostatic pressure also explains that during inversions pressure and blood flow increase in the chest and head along with decreased resistance to draining into the great veins leading to the heart's right atrium. In other words, venous return (preload) is augmented due to gravity when the legs are above chest height. Previously, we conducted a case study (Figure 1) to evaluate real time physiological changes and responses to Hatha yoga inversion and standing postures as a control to determine $\mathrm{VO}_{2}$ and $\mathrm{BP}$ while wearing a wearable metabolic device. Following the protocol of the case study, we conducted a more 
robust clinical study to evaluate real time physiological changes among a group of healthy volunteers (Conroy et al., 2019).

\section{Cardiovascular Effects of Inversion}

There are few published studies on the intersection of yoga and cardiopulmonary physiology of body inversion. Generally, yoga physiology research papers have focused on either cardiovascular or pulmonary measurements in disease states, this research paper examined both simultaneously in normal subjects. Several discordant measurements appear in the findings of cardiovascular measurements with inversion postures. Heng et al., (1992) reported a significant increase in HR during inversion relative to standing, while other researchers have reported a decrease (deVries \& Cailliet, 1985; Sfakianos et al., 1985) or no change in HR (Cardus et al., 1984; Jenning et al., 1985; London et al., 1983). Research on cardiac output (CO) response to gravity inversion is conflicting. Cardus et al. (1984) and Haennel et al. (1988) reported an increase whereas, Heng et al., (1992) reported a decrease. A decrease in systolic blood pressure (SBP) was reported by Wilkins et al. (1950), while Klatz et al., (1983 \&1985; LeMarr et al., 1983) reported an increase or no change during inversion as cited in Haennel et al. (1988). Clearly, subjects in different studies responded differently under a variety of circumstances and protocols. Additionally, the variety of apparatus utilized to measure inversion responses accounts for some of the physiology differences. More recently, Papp et al. (2013) performed a small-scale longitudinal study in naive yoga practitioners, where inversion time increased from seven minutes to 20 minutes over eight weeks and found increased heart rate variability (HRV). This implies that yoga practice can have a beneficial effect on the autonomic nervous system.

With primary focus on the body, breath and mind, yoga is psychophysical in character. Focusing on one's own breathing while practicing yoga body movements, and vice versa, can function as a tool to increase awareness of tension/relaxation states. Thus, increased awareness of body orientation in space is an effective biofeedback instrument. Many of the slow movements in yoga relate to natural 
synchronization between breathing and moving (flow yoga) which promotes slower, deeper and more even paced breathing. In turn, this induces parasympathetic nerve activity and a feeling of relaxation, which can influence HR, BP and breathing pace (Bernardi et al., 2001). Supine and inverted body postures stimulate the baroreceptor reflex (from altered negative pressure in the upper body) and may create parasympathetic (vagal) activity, while upright postures inhibit it. The baroreceptor reflex is involved with HR regulation and closely links to the PNS (Selvamurthy et al., 1998; Cole, 1989).

\section{Pulmonary Effects of Inversion}

The physiology of the cardiopulmonary system is well known. The lungs linked in series with heart circulation are mechanically influenced by the dynamics of cardiac contraction and by cytokines and neuro-humoral modulators involved in cardiopulmonary functions. In a 3-month, randomized controlled study of yoga's effect on pulmonary function parameters, Yadav et al. (2015) studied pulmonary responses in coronary artery disease (CAD) patients and included several supine and prone postures. They reported statistically significant improvement in most of the pulmonary parameters measured, including forced vital capacity $(F V C)$, forced expiratory volume in one second $\left(F^{\prime} V_{1}\right)$, peak expiratory flow rate (PEFR), and maximum voluntary ventilation (MVV). Yadav proposed that reduction of sympathetic reactivity attained with yogic training facilitates broncho-dilatation by correcting the abnormal breathing patterns and reducing the muscle tone of inspiratory and expiratory muscles. Due to improved breathing patterns, respiratory bronchioles may be relaxed and perfusion of alveoli carried out more efficiently (Abel, 2013).

From standing to a supine position, there is only a slight decrease in total lung capacity, residual volume, and vital capacity, but with a large decrease in expiratory reserve volume without an increase in inspiratory reserve volume (Attinger et al., 1956). Pulmonary mixing was diminished in the supine compared to the erect position (Blair \& Hickam, 1955). Changes in hydrostatic pressure and gravity 
influence the physiology of the lung directly by influencing perfusion-ventilation exchange and the distribution of pulmonary blood flow.

The purpose of this study was to address some of the discordant results with yoga inversion in the literature and to consider specific benefits to heart failure patients needing low $\mathrm{VO}_{2}$ demand restorative exercise.

\section{Methods}

The University of North Georgia's (UNG) Institutional Review Board (IRB) approved the study, effective 12/06/2018 with Protocol Code: 2018-196. This study was expanded from the initial case study

Figure 2.

Headstand wearing metabolic device

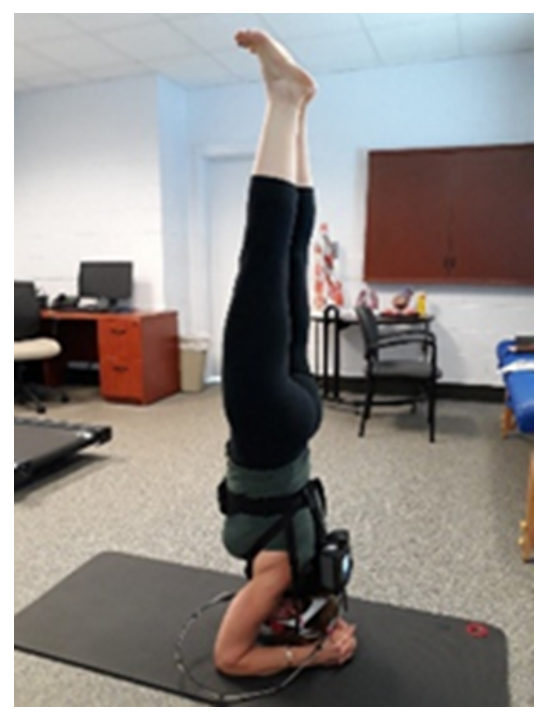

to a more robust clinical study by amending the protocol for $10-20$ participants, approved by UNG-IRB 2020. A convenience sample from a population of yoga students that regularly attended lyengar yoga classes at a yoga studio in Atlanta, Georgia were invited to participate in the study. Inclusion criteria was minimum of two years of yoga practice, including inversions. A portable metabolic device (Cosmed K5), calibrated according to manufacturer's specifications, was worn for the duration of each trial (Figure 2).

A yoga teacher (RYT ${ }^{\circledR} 500$-Yoga Alliance) led each participant

in a sequence of postures that included whole body inversions. Each half-hour trial followed a specific protocol (Appendix A) with the order between inversion and standing postures alternating. Brachial BP was obtained using an AlphaMed fully automatic blood pressure monitor (Model U80R) during the last 30 seconds of each posture. Heart rate, $\mathrm{VO}_{2}$ and Respiratory Quotient (RQ) were recorded every 10 seconds by the metabolic device. The trial began with a two-minute crossed leg pose, followed by a warm-up consisting of breathing exercises and a dynamic sequence of postures. The second sequence included Tadasana (mountain pose) then Utkatasana (chair pose) held for two minutes each. For the 
third sequence, Viparita Karani and Sirsasana were performed in place of the standing postures, each for four minutes and ended with Savasana (corpse pose) for relaxation (see protocol, Appendix A).

Analysis followed the sequence $A->B->C$ corresponding to standing $(A)$, supine $(B)$ and inverted (C) postures. Related cardiovascular responses were calculated according to the formulas below (Koenig et al., 2015):

(1) Stroke volume (SV) calculated by dividing pulse pressure (SBP-DBP) by (SBP+DBP)

(2) Cardiac output equals SV x HR

(3) Arteriovenous oxygen difference calculated by dividing oxygen uptake by $\mathrm{CO}$

(4) Mean arterial pressure (MAP) calculated using the formula: $\mathrm{MAP}=\mathrm{DBP}+.32(\mathrm{SBP}-\mathrm{DBP})$

(5) Systemic vascular resistance (SVR) calculated by dividing MAP by CO

Statistics: Student's t-test determined significance of change in the cardiovascular responses between postures $A->B, A->C, B->C$, or $A->C$. The alpha level was set at 0.05 for statistical significance.

\section{Results}

Twelve yoga practitioners (mean age 44 years, 58\% female) participated in 17 trials recording changes in $\mathrm{BP}, \mathrm{VO}_{2}, \mathrm{RQ}$ and related $\mathrm{METs}$ (Table 1). Systolic blood pressure ranged from $152 \mathrm{mmHg}$ for Sirsasana to $125 \mathrm{mmHg}$ for Savasana, while the associated diastolic blood pressure (DBP) ranged from 103 to $77 \mathrm{mmHg}$. A t-test between meditation and inversion for $\mathrm{VO}_{2}$ and $\mathrm{HR}$ was significant $(\mathrm{p}<0.05)$. As

\section{Table 1}

Comparisons Across Yoga Postures

\begin{tabular}{lllllll} 
Yoga Pose & $\mathrm{SBP}$ & $\mathrm{DBP}$ & $\mathrm{RQ}$ & $\mathrm{VO}_{2}$ & $\mathrm{MET}$ & $\mathrm{HR}$ \\
\hline Sirsasana & 151.8 & 103.2 & 0.91 & 8.44 & 2.41 & 80.9 \\
Utkatasana & 140.4 & 94.4 & 0.85 & 10.14 & 2.90 & 104.9 \\
Warm-up & 136.9 & 87.8 & 0.81 & 11.53 & 3.29 & 99.0 \\
Tadasana & 136.2 & 88.4 & 0.99 & 7.59 & 2.17 & 84.2 \\
Study Start & 132.4 & 83.5 & 0.78 & 5.41 & 1.55 & 74.4 \\
Pranayama & 131.0 & 85.8 & 0.80 & 4.40 & 1.24 & 75.1 \\
Viparita Karani & 130.2 & 78.4 & 0.94 & 5.31 & 1.52 & 67.9 \\
Savasana & 125.2 & 76.8 & 0.95 & 4.90 & 1.42 & 71.8
\end{tabular}

Notes. Prana = Pranayama-breathing exercises; Viparita Karani or legs up the wall (a supported inversion). Savasana= resting or corpse pose; $\mathrm{SBP}=$ systolic blood pressure; $\mathrm{DBP}=$ diastolic blood pressure; $\mathrm{RQ}=$ respiratory quotient; $\mathrm{VO}_{2}$ as relative oxygen consumption in $\mathrm{ml} / \mathrm{kg} / \mathrm{min}$; $\mathrm{METs}=$ metabolic equivalents; $\mathrm{HR}=$ heart rate in beats per minute. 
with another study (Heng et al., 1992), BP was elevated during inversion. Our initial case study (Conroy et al., 2019) found a mean $\mathrm{VO}_{2} / \mathrm{kg}$ of $3.1 \pm 0.08$ for the meditative pose, while the inverted poses yielded a mean $\mathrm{VO}_{2}$ of $19.5 \pm 1.5 \mathrm{ml} / \mathrm{kg} / \mathrm{min}$. A RQ of less than 0.8 indicates primarily fat utilization for energy, so the participants arrived at the trial burning fat stores; while during the trial, carbohydrate metabolism dominated ATP production. Oxygen consumption or METs corresponded roughly with SBP. The highest energy pose was the warm-up phase at 3.1 METs, followed by the difficult Utkatasana and closely behind was Sirsasana at 2.4 METs. Heart rate generally followed SBP. Heart rate response ranged

\section{Table 2}

Statistical comparisons between yoga poses for primary variables

\begin{tabular}{|l|l|l|l|l|}
\hline Yoga Posture & $\mathrm{SBP}$ & $\mathrm{DBP}$ & $\mathrm{VO}_{2}$ & $\mathrm{HR}$ \\
\hline Viparita Karani & 131 & 80 & 5.3 & 70 \\
\hline Sirsasana & 154 & 105 & 8.3 & 82 \\
\hline Compare 2C t-test & 0.071 & 0.005 & 0.000 & 0.000 \\
\hline Tadasana & 131 & 87 & 7.4 & 84 \\
\hline Sirsasana & 152 & 110 & 9.1 & 82 \\
\hline Compare A-C t-test & 0.101 & 0.004 & 0.001 & 0.569 \\
\hline Utkatasana & 131 & 91 & 10.4 & 104 \\
\hline Sirsasana & 155 & 108 & 8.6 & 78 \\
\hline Compare A->Ctest & 0.100 & 0.159 & 0.032 & 0.000 \\
\hline Savasana & 127 & 73 & 8.3 & 81 \\
\hline Viparita Karani & 130 & 78 & 5.3 & 68 \\
\hline Compare B->C t-test & 0.735 & 0.035 & 0.000 & 0.000 \\
\hline Savasana & 121.2 & 74.7 & 5.0 & 70.3 \\
\hline Sirsasana & 159.4 & 107.7 & 8.3 & 81.5 \\
\hline Compare B->C t-test & 0.009 & 0.002 & 0.001 & 0.002 \\
\hline Tadasana & 130.5 & 85.6 & 7.4 & 83.4 \\
\hline Viparita Karani & 126.9 & 76.7 & 5.7 & 66.7 \\
\hline Compare A->C t-test & 0.296 & 0.049 & 0.002 & 0.000 \\
\hline Study Start & 129 & 84 & 8.4 & 79 \\
\hline Savasana & 125 & 76 & 5.4 & 72 \\
\hline Compare B to Start t-test & 0.564 & 0.044 & 0.000 & 0.008 \\
\hline P<O.05 & & & & \\
\hline & & & & \\
\hline
\end{tabular}

Note. $\mathrm{SBP}=$ Systolic blood pressure; $\mathrm{DBP}=$ Diastolic blood pressure; $\mathrm{HR}=$ Heart rate; $\mathrm{VO}_{2}=\mathrm{ml} / \mathrm{min} / \mathrm{Kg}$; Paired t-test of comparisons between positions A standing, B supine, and C inverted. Parameter means can be different due to some missing values that occurred during some trials. 
from 105 beats per minute (bpm) for Utkatasana to Savasana (resting) at 72 bpm.

Over all trials, mean oxygen consumption for Sirsasana as compared with supported inversion posture decreased from 8.4 to $4.9 \mathrm{ml} / \mathrm{kg} / \mathrm{min}$. Significance of comparing various poses on body position transitioning from standing to inverted are shown in Table 2. A t-test between Sirsasana and Viparita Karani for $\mathrm{VO}_{2}$ consumption, $\mathrm{HR}$, and DBP were significantly different $(\mathrm{p}<0.005)$. All variables in Table 2 comparing supine resting (Savasana) and Sirsasana, were significantly different $(p \leq 0.009)$. There are also significant differences for all values except SBP, between Tadasana and Karani, as well as study start and Savasana $(p<0.05)$. Comparison of Utkatasana to Sirsasana pose follows the sequence of standing to inversion $(A->C)$, while Tadasana/Savasana/ Viparita Karani follows the sequence of standing/ supine/inversion ( $\mathrm{A}->\mathrm{B}->\mathrm{C})$. Comparison t-tests were run paired, so the means shown in Table 2 are often.

Measurements of $\mathrm{CO}$ and other derived hemodynamic variables obtained from $\mathrm{HR}$ and blood pressures (SBP and DBP) shown in Table 3. Comparisons of physiological responses to standing, supine and supported inversion were made between the current and previous studies (Boone, 2002; Heng et al., 1992). Heart rate emerged as the

\section{Table 3}

Three Body Positions Comparison with Literature Trends

\begin{tabular}{|l|l|l|l|l|l|l|l|l|l|}
\hline $\begin{array}{l}\text { Body } \\
\text { position }\end{array}$ & SBP & DBP & $\mathrm{HR}$ & $\begin{array}{l}\mathrm{VO}_{2} \\
\mathrm{~mL} / \mathrm{Kg} / \mathrm{min}\end{array}$ & $\mathrm{RPP}$ & $\mathrm{SV}$ & $\mathrm{CO}$ & $\mathrm{MAP}$ & $\mathrm{SVR}$ \\
\hline Tadasana & 136 & 88 & 84 & 7.59 & 11582 & 0.21 & 17.2 & 103.7 & 6.01 \\
\hline Savasana & 125 & 77 & 72 & 4.90 & 8987 & 0.24 & 16.8 & 92.3 & 5.51 \\
\hline $\begin{array}{l}\text { Viparita } \\
\text { Karani }\end{array}$ & 130 & 78 & 68 & 5.31 & 8922 & 0.25 & 16.9 & 95.0 & 5.62 \\
\hline Trends & & & & & & & & & \\
\hline This Study & 0 & $\downarrow$ & $\downarrow$ & $\downarrow$ & $\downarrow$ & 0 & 0 & $\downarrow$ & 0 \\
\hline Boone & 0 & & $\downarrow$ & 0 & & $\uparrow$ & $\uparrow$ & 0 & $\downarrow$ \\
\hline Heng & $\uparrow$ & $\uparrow$ & $\uparrow$ & & $\uparrow$ & & $\downarrow$ & & $\uparrow$ \\
\hline
\end{tabular}

Notes. Numeric values in black are actual measurements; numbers in italics are derived values. Trends are for standing, supine, and inverted orientations: $\uparrow=$ increase, $0=$ statistically no change, $\downarrow=$ decrease. For $\underline{\text { This Study }}$ row, at least 2 out of the three position transitions must be significant $(A->B, A->, B-C)$ to determine a trend. 
variable that decreased going from standing to supine to inversion for both this study and Boone's study. Within this study, BP, HR, VO2, rate pressure product and MAP decreased significantly going from standing to inversion whereas, SBP, SV, CO and SVR remained unchanged.

\section{Yoga Exergames and Energy Expenditure}

Exergaming is a term used for video games that are also a form of exercise. Exergaming uses technology that tracks body movement, which has upending the stereotype of gaming as a sedentary activity. Systems such as Xbox use input devices like the Kinect sensor that measures and scores movement then superimpose animated objects or avatars to interact with over a video image of the user (Pullen \& Seffens, 2018). In order to estimate energy expenditure during an exergame yoga session, we earlier measured $\mathrm{O}_{2}$ consumption rates and METs for selected yoga postures (Table 4) using a Parvo Medics TrueOne 2400 metabolic cart (Pullen et al., 2015). Setup and calibration were conducted as detailed in operating manual. We measured $\mathrm{O}_{2}$ consumption for a series of yoga positions as mentioned previously (ibid.). As expected, more intense positions have higher energy consumption. Difference between the average MET for Tadasana in Table 4 (at 1.46) with the value of 2.0 in Table 1 is probably due to the different metabolic carts (Parvo Medics vs Cosmed K5) utilized between these studies.

\section{Table 4}

Metabolic Equivalents (METs) for Yoga Postures

\begin{tabular}{llllllll}
\multicolumn{3}{l}{ Subject } & Gender Age & \multicolumn{3}{l}{ Standing Mountain Arms Up Warrior Rev-W } \\
\hline 1 & F & 57 & 0.95 & 1.55 & 1.5 & 1.8 & 2.3 \\
2 & M & 61 & 1.03 & 1.15 & 1.35 & 2.05 & 2.35 \\
3 & M & 21 & 1.32 & 1.1 & 1.7 & 2.25 & 2.55 \\
4 & F & 21 & 1.35 & 2.05 & 1.9 & 1.95 & 2.65 \\
5 & M & 26 & 1.07 & 1.2 & 1.15 & 1.75 & 2 \\
6 & M & 21 & 1.5 & 1.75 & 1.25 & 2.9 & 3.8 \\
\hline AVG & M & 34.5 & 1.2 & 1.46 & 1.48 & 2.12 & 2.61
\end{tabular}

Note. Standing is relaxed; Mountain is Tadasana which engages postural support muscles; Warrior is Virabhadrasana I; Rev-W is Viparita Virabhadrasana (Pullen, et al., 2015). 


\section{Discussion}

Consistent with Boone (2002), cardiac output increased with supine versus standing and during the inverted versus supine and standing, although not statistically significant. Increased CO directly related to a reduction in the effects of gravity in standing and seated positions, may promote blood pooling in the lower extremities. Since the supine position effectively neutralizes gravity effects on the cardiovascular system, the transition from standing to supine presumably increases venous return, enddiastolic volume, and therefore stroke volume (SV in Table 3). With subjects in the inverted position, the effect of gravity should also increase venous return. Since the subjects' HR decreased from standing to supine (transition $\mathrm{A}->\mathrm{B}$ ) and inverted positions (transition $\mathrm{B}->\mathrm{C}$ ), and yet CO remained nearly constant as shown in Table 3, then stroke volume correspondently increased.

The paucity and diversity of scientific studies in the literature concerning body inversion, specific to the practice of yoga postures highlights the need for future research. Heng et al., (1992) reported a significant increase in HR during inversion relative to standing, while other researchers have reported a decrease (Table 3 in this study) or no change in HR. The CO response to inversion in the present study remained the same. Similarly, systolic blood pressure (SBP) previously reported to increase or decrease or did not change significantly with inversion (Haennel et al., 1988). The variety of protocols and circumstances these few studies reported, may be explained by the different protocols and participant experience with inversion and highlights the need for protocol standardization and clinically specific population studies. For these reasons, comorbidities such as, hypertension, history of stroke or heart disease warrants a cautious approach when engaging with inversion postures. Our previous study with HF patients did include Viparita Karani with BP checks without adverse events (Pullen et al., 2010).

Boone's study (2002) of fourteen healthy subjects used a gravity-inversion apparatus with the subjects standing upright for five minutes. Resting BP and HR were measured after four minutes while CO was measured at the fifth minute. This was repeated for the horizontal pose (supine B position) and 
subject was rotated to a 45 -degree head-down position for the final five minutes. Transitioning from standing to supine $(A->B)$ to head-down $(B->C)$ did not alter $\mathrm{O}_{2}$ consumption nor mean arterial pressure. Cardiac output and SV increased in supine position (A->B) and increased further with the inversion (B$>C$ ) positioning. Heart rate decreased during supine and inverted positions. Systemic vascular resistance decreased during the supine position $(A->B)$ and decreased further during the inverted position (B->C). Systolic blood pressure increased from inversion relative to standing and supine. Boone concluded that $\mathrm{O}_{2}$ consumption was not altered depending on whether they were standing, supine, or in an inverted position. With $\mathrm{VO}_{2}$ unchanged, the increase in $\mathrm{CO}$ was balanced by a decrease in extraction of oxygen by the body.

\section{Adverse Events with Yoga Inversions}

Yoga is regarded as beneficial to most people, yet the safety of yoga has been questioned in some popular-press articles, prompting a systematic review by Cramer et al., (2013) that included 76 cases of adverse events associated with yoga. Sirsasana and Salamba Sarvangasana (supported shoulder stand) were the most often-cited yoga postures associated with adverse events. Yoga instructors, assumed to practice more intensely than yoga students do, have reported adverse events. Two of the three cases who had practiced Salamba Sarvangasana and eight of the ten cases that had practiced Sirsasana reported orbital adverse events, mainly glaucomatous symptoms (ibid.). While there are reports that Sirsasana induces a twofold increase in intraocular pressure, values returned to baseline promptly afterwards, and association of regular yoga practice with chronically increased intraocular pressure was not found (Baskaran et al., 2006).

Little evidence exists regarding the relationship between inversions and other pre-existing conditions. Therefore, all yoga students with comorbities should be cautious with inversions, which may be contraindicated for individuals with a history of glaucoma. As with any other physical or mental practice, yoga is not without possible risk. Given the large number of yoga practitioners worldwide, 
relatively few serious adverse events have been reported. The conclusion of a systematic review and meta-analysis of yoga for chronic disease management was that the practice of yoga significantly improved exercise capacity and reported quality of life (Desveaux, Lee, Goldstein \& Brooks, 2015).

\section{Conclusion}

Physiological responses varied between mediation Hatha yoga, inversion (experimental) and standing postures (control). Reliability and validity of portable $\mathrm{VO}_{2}$ and rate pressure product measurements made during yoga postures were examined in a cohort of experienced yoga practitioners. These results add to broader aspects of yoga and physiological measurements discussed above with further trials and additional subjects planned. This platform of inquiry may be utilized for teaching physiology to kinesiology students and assist in implementation of yoga and other meditative movement technologies.

Evidence of discordant findings exist in the literature concerning physiology of inversion. The CO response to inversion has been reported as increased or decreased. Participants responded differently under a variety of protocols in previous studies, making comparisons to existing research challenging. We recommend further research on the physiological response to supported inversion postures with cohorts of targeted populations such as heart failure patients be investigated for therapeutic outcomes.

\section{Conflict of Interest to Declare}

The authors have no conflicts of interest to disclose.

\section{Statement of Funding}

This study was supported in part by an intramural CURCA grant from UNG and technical support from Seftec, Inc. We wish to acknowledge the support Professor Jason Casey at UNG and Kathleen Pringle owner of Stillwater Yoga Studio, who supported the study by sharing her studio space and yoga students. 
Physiology during Yoga Inversion

\section{References}

Abel, A.N., Lloyd, L.K., Williams, J.S. (2013). The effects of regular yoga practice on pulmonary function in healthy individuals: a literature review. Journal of Alternative and Complement Medicine. 19(3):185-190. doi:10.1089/acm.2011.0516

Attinger, E.O., Monroe, R.G., \& Segal, M.S. (1956). The Mechanics of Breathing in Different Body Positions. I. In Normal Subjects. Journal of Clinical Investigation. 35(8), 904-911.

Barach, A.L., \& Beck, G.J. (1954). The ventilatory effects of the head-down position in pulmonary emphysema. American Journal of Medicine, 16, 55-60.

Baskaran, M, Raman, K, Ramani K.K., Roy, J., Vijaya, L., Badrinath, S.S. (2006). Intraocular pressure changes and ocular biometry during Sirsasana (headstand posture) in yoga practitioners. Ophthalmology, 113, 1327-1332. DOI: 10.1016/i.ophtha.2006.02.063

Bernardi, L., Gabutti, A., Porta, C., \& Spicuzza, L. (2001). Slow breathing reduces chemoreflex response to hypoxia and hypercapnia, and increases baroreflex sensitivity. Journal of Hypertension, 19, 2221-2229. doi: 10.1097/00004872-200112000-00016

Blair, E., \& Hickam, J. B., (1955). The effect of change in body position on lung volume and intrapulmonary gas mixing in normal subjects. Journal of Clinical. Investigation, 34(3), 383-389.

Blomqvist, C.G., Nixon, J.V., Johnson, R.J., \& Mitchell, J.H. (1980). Early cardiovascular adaptation to zero gravity simulated by head-down tilt. Acta Astronautica, 7, 543-553.

Boone, T. \& Johns, K. (1989). Cardiorespiratory and hemodynamic responses to inversion and inversion with sit-ups. Journal of Sports Medicine and Physical Fitness, 29(4), 346-357.

Boone, T. (2002). Gravity Inversion: A Teaching Tool for Integrating Critical Thinking and Cardiovascular Physiology, Professionalization of Exercise Physiology online, 5(11). https://www.asep.org/asep/asep/ThinkingOutsideTheBoxExercisePhysiology.html 
Physiology during Yoga Inversion

Cardus, D., McTaggart, W.G., \& Domingo, E. (1984). Cardiovascular changes with head-down ankle suspension. The Physiologist, 27, 45-46.

Cole, R.J. (1989). Postural baroreflex stimuli may affect EEG arousal and sleep in humans. Journal of Applied Physiology. 67(6), 2369-2375.

Conroy, K-B., Casey, J., Seffens, W., \& Seffens, P. (2019). Case Study of Physiological Measurements during Yoga Asana Practice. Medicine \& Science in Sports \& Exercise, 51(6S):258 doi: 10.1249/01.mss.0000561281.80782.36

Cramer, H., Krucoff, C., \& Dobos, G. (2013). Adverse Events Associated with Yoga: A Systematic Review of Published Case Reports and Case Series. PLoS ONE 8(10): e75515. doi:10.1371/journal.pone.0075515

deVries, H.A., \& Cailliet, R. (1985). Vagotonic effect of inversion therapy upon resting neuromuscular tension. American Journal of Physical Medicine, 64, 119-129.

Haennel, R.G., Teo, K.K., Snydmiller, G.D., Quinney, H.A., \& Kappagoda, C.T. (1988). Short-time cardiovascular adaptations to vertical head-down suspension. Archives of Physical Medicine and Rehabilitation, 6, 352-357.

Heng, M. K., Bai, J. X., Talian, N. J., Vincent, W. J., Reese, S. S., Shaw, S., \& Holland, G. J. (1992). Changes in cardiovascular function during inversion. International Journal of Sports Medicine, 13(1), 69-73.

Jenning, T., Seaworth, J., Howell, L., Trip, L., \& Goodyear, C. (1985). Effect of body inversion on hemodynamics determined by two dimensional echocardiography. Critical Care Medicine Journal, 13:760-762.

Klatz, R.M. (1983). Gravity Inversion Boots--A Potential Hazard, British Journal of Sports Medicine, 17(4), 154-155. 
Klatz, R.M., Goldman, R.M., Pinchuk, B.G., Nehon, K.E., \& Tarr, R.S. (1983). Effects of gravity inversion procedures on systemic blood pressure, intraocular pressure, and central retinal arterial pressure. Journal of the American Osteopathic Association, 82, 853-857.

Klatz, R.M., Goldman, R.M., Pinchuk, B.G., \& Tarr, R.S. (1985). Effects of gravity inversion on hypertensive subjects. Physician and Sportsmedicine, 13, 85-89.

Koenig, J., Hill, L.K., Williams, D.P., \& Thayer, J.F. (2015). Estimating Cardiac Output from Blood Pressure and Heart Rate: The Liljestrand \& Zander Formula. Biomedical Sciences Instrumentation, 51:85-90.

Larson-Meyer, D.E. (2016). A systematic review of the energy cost and metabolic intensity of yoga. Medicine \& Science in Sports Medicine, 48, 1558-1569.

LeMarr, J. D., Golding, L. A., \& Crehan, K. D. (1983). Cardiorespiratory responses to inversion. The Physician and Sports Medicine, 11(11), 51-57.

London, G.M., Levenson, J.A., Safar, M.E., Simon, A.C., Guerin, A., Payen, D. (1983). Hemodynamic effects of head-down tilt in normal subjects and sustained hypertensive patients. American Journal of Physiology, 245, 194-202.

Papp, ME, Lindfors, P, Storck, N. \& Wändell, PE (2013). Increased heart rate variability but no effect on blood pressure from 8 weeks of hatha yoga - a pilot study. BMC Research Notes, 6, 59.

Pullen, P., Ogbesor, A., \& W Seffens (2015). Kinect Acquisition of skeleton body positions during yoga and tai chi for exergame development. Medicine \& Science in Sports \& Exercise, 47 5S, 162 doi: 10.1249/01.mss.0000476856.07206.5e

Pullen, P.R., Thompson, W.R., Benardot, D., Brandon, J.L., Mehta, P.K., Rifia, L., Vadnais, D.S., Parrott, J.M., \& Khan, B.V. (2010). Benefits of Yoga for African American Heart Failure Patients. Medicine \& Science in Sports \& Exercise, 42(4), 651-657. 
Physiology during Yoga Inversion

Pullen, P. \& Seffens, W. (2018). Machine learning gesture analysis of yoga for exergame development. Special issue Healthcare applications in Cyber-Physical Systems Journal, http://digital-library.theiet.org/docserver/fulltext /10.1049/iet-cps.2017.0027/IETCPS.2017.0027.pdf

Pullen, P.R., Seffens, W., \& Thompson, W.R. (2018). Yoga for Heart Failure Patients: Current Status, International Journal of Yoga, 11(2), 91-98.

Selvamurthy, W., Sridharan, K., Ray, U.S., Tiwary, R.S., Hegde, K.S., Radhakrishan, U., Sinha, K.C. (1998). A new physiological approach to control essential hypertension. The Indian Journal of Physiology and Pharmacology, 42(2), 205-213.

Sfakianos, P.N., Hargens, A.R., \& Akeson, W.H. (1985). Microvascular flow adjustments with postural changes in humans. The Physiologist, 28, 175-176.

Wilkins, R.W., Bradley, \& S.E., Friedland, C.K. (1950). The acute circulatory effects of the headdown position on normal man, Journal of Clinical Investigation, 29, 940-949.

Yadav, A., Singh S, Singh K, Pai, P. (2015). Effect of yoga regimen on lung functions including diffusion capacity in coronary artery disease patients: A randomized controlled study. International Journal of Yoga, 8(1), 62-67. doi:10.4103/0973-6131.146067

Yoshikawa, Y. (2017). Everybody Upside-Down. Yoga Journal. April 13. https://www.yogajournal.com/practice/everybody-upside-down 


\section{Appendix}

*Yoga Inversion Study Protocol: Physiological Measurements during Yoga@ by Dr. Paula Pullen (aka Seffens)

Subject ID: Date: Weight: lbs. $/ 2.2=$ kg Height: $\mathrm{cm}$ $\mathrm{M}$ or $\mathrm{F}$

\section{PRE-TRIAL ACTIVITY}

$\square K 5$ warm-up for $20 \mathrm{~min}$. $\square$ K5 Calibrated $\square \mathrm{VO}_{2}$ Mask Set-Up $\square$ HR monitor on participant $\square$ K5 fitted $\square$ Participant seated on floor in comfortable position (support as needed in cross leg pose-Sukasana) $\square$ Start K5 and allow $2 \mathrm{~min}$ for calibration (after $2 \mathrm{~min}$, time will restart at 0:00) TRIAL

$\square$ Start time on $\mathrm{K} 5$ clock: (Use stopwatch to measure individual time segments)

Baseline: BP on Right Arm then Left Arm: (R) $\mathrm{mm} \mathrm{Hg}$

(L) $\mathrm{mm} \mathrm{Hg}$

[Standardize all BPs to left arm unless $>10 \mathrm{~mm}$ difference noted at start which is a study exclusion criteria.]

\section{PRANAYAMA \& ASANA- WARM-UP (SEQUENCE ONE)}

$\square$ Start 3 rounds of breathing exercise while seated (Pranayama- equal inhale \& exhale duration)

$\square$ Start time on $\mathrm{K} 5$ clock

Post-Pranayama BP: $\mathrm{mm} \mathrm{Hg} \quad$ Time B/P obtained

From Tadasana (standing Mountain pose)

$\square$ Start 3 rounds moving from Urdhva Hastasana (Upward Salute) to Uttanasana (standing forward bend) to Adho Mukha Svanasana (Downward-Facing Dog Pose)

$\square$ Start time on $\mathrm{K} 5$ clock

$\square$ BP immediately upon stopping:

1 $\mathrm{mm} \mathrm{Hg} \quad \square$ End Time on $\mathrm{K5}$ clock:

ASANA POSTURES (SEQUENCE TWO)

1. Utkatasana (Chair Pose- Arms above Head) - 2 min $\square$ Start Time on K5 clock:

$\square$ Begin BP during last 30 seconds: BP ___ $\mathrm{mm} \mathrm{Hg} \square$ End Time on $\mathrm{K5}$ clock:

2. Tadasana (Mountain Pose) -2 min $\square$ Start Time on $\mathrm{K5}$ clock:

$\square$ Begin BP during last 30 seconds: BP: $\mathrm{mm} \mathrm{Hg} \square$ End Time on $\mathrm{K5}$ clock: NVERSIONS (SEOUENCETHREE)

1. Sirsasana (Head stand) -4 min

$\square$ Start Time on $\mathrm{K5}$ clock:

$\square$ BP one: start at minute $1^{\prime} 30^{\circ}$

$\square$ BP two: start at minute $3^{\prime} 30^{\circ}$

$\square$ End Time on $\mathrm{K} 5$ clock:

2. Viparita Karani - (Legs on the wall- hips supported by a 4-5 inch thick bolster) - 4 min

$\square$ BP one: start at minute $1^{\prime} 30^{\prime \prime}$ _ $\square$ BP two: start at minute $3^{\prime} 30^{\prime \prime}$ 1

\section{RELAXATION (SEQUENCE FOUR)}

$\square$ Savasana (corpse pose) -5 minutes

Start time on $\mathrm{K} 5$ clock:

$\square$ Begin BP in last 30 seconds: Min 2 BP: $\mathrm{mm} \mathrm{Hg} \quad \square$ End Time on $\mathrm{K} 5$ clock:

\section{Stop the $\mathbf{K} 5$ clock!}

